\title{
Modelling a Hybrid Wireless/Broadband over Power Line (BPL) Communication in $5 \mathrm{G}$
}

\author{
Mohammad Woli Ullah', Mohammad Azazur Rahman², Md. Humayun Kabir ${ }^{3}$, Muhammad Mostafa Amir Faisal ${ }^{4}$ \\ Dept. of Electronic and Telecommunication Engineering \\ International Islamic University Chittagong \\ Chittagong, Bangladesh
}

\begin{abstract}
G will explore wireless communication dynamically, which will provide high-speed internet with low latency. So, real-time communication will be possible, and a vast number of devices will connect from different points. 5G has introduced five enabling technologies. Millimeter wave and small cell are two of them. Small cell connects the user devices by using the millimeter wave. As the frequency range has limitations of distance and high attenuation, it should be reliable for uninterrupted communication. To ensure the uninterrupted communication, hybrid communication network can be a significant solution. In this research, hybrid wireless and Broadband over Power Line (BPL) communication model has been proposed, and the model integrates both technologies in the small cell end. A simulation of BPL and theoretical analysis of wireless communication have also been shown in this paper. From those analysis, the total throughput of the hybrid model has been calculated. Broadband over Power Line is chosen as well as wireless communication in this model because of its infrastructure availability both in city and rural areas, costeffectiveness and quick installation process. Moreover, the hybrid network will increase the throughput volume, and both communications will act as a backup in an emergency.
\end{abstract}

Keywords-5G; wireless; broadband over power line; hybrid; millimeter wave; small cell

\section{INTRODUCTION}

$5 \mathrm{G}$ is the next generation high speed wireless mobile communication technology that will integrate various technologies. It will not only provide high-speed internet but also serve a large amount of bandwidth. 5G will be a hundred times faster than $4 \mathrm{G}$ mobile communication [1]. As a result, real-time communication will be possible. The autonomous vehicle, augmented reality, highly intelligent robot and other technologies will integrate with $5 \mathrm{G}$ [1], and all the devices will work rapidly.

Millimeter-wave is a significant aspect of 5G. $5 \mathrm{G}$ will use high-frequency bandwidth for data transmission, and the bandwidth range is $30-300 \mathrm{GHz}$ [1]. So, the latency will decrease than $4 \mathrm{G}$, and the user will get a vast amount of throughput. It is called mm-Wave as the wavelength of frequency in $5 \mathrm{G}$ is $10-1 \mathrm{~mm}$ [1] and able to serve $10-100 \mathrm{Gbps}$ data speed theoretically.

However, it has some demerits also. Millimeter-wave is not suitable for long-distance communication [1]. It can cover about $300 \mathrm{~m}$ area [2]. It has high attenuation and fading. The signal is distracted by building, trees, and other obstacles [1].
To solve this problem, small cell technology has been introduced. A large number of small cells will connect with a Base Transceiver Station (BTS). It will be easy to radiate mmwave to the end devices with low attenuation. The small cell will be placed near the user, and it will be about 300m [2] away. It will work as a $\mathrm{Wi}-\mathrm{Fi}$ router and can be placed on electrical poles, rooftops, trees, and other places, from where mmWave can reach to end device. When device becomes mobile, small cells will hand over the network operation to another nearest small cell. The small cell carries a massive MIMO antenna. So, multiple number of users can connect with a small cell simultaneously and ensure continuous data transfer.

\section{BACKGROUND OF THE WORK}

As small cell will be placed near to end-user, it will be possible to provide better throughput with continuation of the signal. However, it has a chance to distract the signaling between BTS and small cells. Weather attenuation, obstacle density, and other resistive issues can occur. To make a reliable uninterrupted communication, researchers have proposed some hybrid communication model. The proposed models have shown the possibility of uninterrupted communication. Hybrid LTE and DSL network has been proposed in [2] to improve the throughput in $4 \mathrm{G}$ communication for Malaysian rural area. However, the hybrid model has been simulated only for the downstream feature. This limitation has been addressed in [3]. In [3], hybrid consumer premises equipment (HCPE) and hybrid access gateway (HAG) have to integrate LTE and DSL. The architecture will provide the upstream and downstream simultaneously in $4 \mathrm{G}$.

Fixed Broadband (FBB) and Mobile Broadband (MBB) were aggregated to provide hybrid network where both technologies are existing [4]. It would increase the throughput capacity in the city area. The research has been focused on some aspects of hybrid network such as traffic aggregation, data rates, latency, and link utilization [4]. However, the hybrid model is not sufficient for the rural area, especially for the rural area of underdeveloped and developing countries. This is because optical fiber network is not available in those rural areas massively. This model can be implemented widely in city and urban areas.

Free space optics (FSO) can be a candidate for hybrid communication in 5G [5]. The technology is suitable in mega cities where there are many high-raise buildings, and it saves 
infrastructure cost of optical fiber [5]. In [5], a hybrid network of wireless mobile and free space optics has been proposed and the network will connect with a cloud RAN. The significant drawbacks of the hybrid model are that both communications depend on the weather. The research paper has been done by using Egyptian weather information. In foggy weather, when the visibility ( $\mathrm{V}$ ) is $\mathrm{V} \geqslant 0.55 \mathrm{~km}$, the hybrid network can reach to $100 \%$. However, at visibility $\mathrm{V}<0.55 \mathrm{~km}$, the network falls down gradually, and the network availability is $0 \%$ at $\mathrm{V}<0.24 \mathrm{~km}$. Moreover, light rain network availability is $100 \%$ for the hybrid network, but it decreases with increment in rain [5]. So, the FSO/mm Wave hybrid network is not reliable for ensuring uninterrupted communication with continuous throughput [5]. Channel estimation of the FSO/mmWave hybrid network has been shown in [6]. The SNR for the dual-channel has been measured here, and it has been demonstrated that strong air turbulence is another limitation for $\mathrm{FSO} / \mathrm{mm}$ Wave hybrid communication.

Satellite and XDSL broadband links have been proposed for hybrid communication to ensure an uninterrupted channel [6]. The simulation architecture for hybrid network has been shown with respect to ISO layers. However, the network setup is not convenient, and throughput will not be up to the mark.

Visible Light Communication (VLC) is a prominent technology for indoor communication. However, it has a lineof-sight (LOS) limitation [1]. VLC and mm Wave wireless technology can establish a hybrid $5 \mathrm{G}$ network for the indoor user, where a hybrid access point (HAP) will control the throughput distribution [8]. A hybrid VLC/mm Wave model and its statistics have been shown in [8].

To ensure high speed internet for indoor purpose, optical and wireless $\mathrm{mm}$ Wave technology can be used combinedly [9]. It will ensure high data rate and energy efficiency. But the network can't be deployed in the rural areas because of lack of infrastructure. The experimental simulation setup and simulation output have been shown in [9].

An overview of different types of the feasible hybrid network for $5 \mathrm{G}$ has been shown in [10]. The hybrid networks are VLC/Microcell, Li-Fi/small cell, FSO/RF, OCC/Wi-Fi, LiFi/Microcell etc. Those networks are compatible with a particular purpose, and it will help to decide proper hybrid network for a different purpose.

In this research, we have also proposed a hybrid network for $5 \mathrm{G}$ coverage. Wireless $\mathrm{mm}$ Wave and Broadband over Power Line (BPL) have been considered as communication methods for hybrid communication. BPL has been considered because of some aspects such as cost efficiency, easy installation, penetration availability in terms of both city and rural area. AT\&T has already taken a project called AirGig, which will deploy 5G by using BPL technology [12]. A hybrid model of the communication system and combined throughput of it has been shown in this paper.

Rest of the paper is organized as follows. In the Section III, importance of hybrid communication is described. Basic components of the model and their explanation are discussed in Sections IV and $\mathrm{V}$, respectively. In the sixth section, the possible throughput of the hybrid model is calculated and the last section summarizes the work with a conclusion.

\section{IMPORTANCE OF HYBRID COMMUNICATION}

\section{A. Communication Reliability}

In wireless $5 \mathrm{G}$ network, so many barriers are there which are a threat to reliable communication. The barriers should be considered in different environments. As millimeter-wave will be used in $5 \mathrm{G}$, signal distortion is a common issue. Moreover, it has other limitations such as the line of sight, user density, propagation loss, attenuation, free space loss, foliage loss, blockage loss, and so more [1]. In that case, Broadband over Power Line can act as the backup reliable communication method. When the particular communication channel won't be able to provide proper throughput, another channel will offer the service to stabilize the data flow. Additionally, hybrid communication will provide the best data rate by both channels to the customers in the general situation. In Fig. 1, the communication reliability has been shown.

\section{B. Bandwidth Coverage}

Wireless Communication can't perform well in inaccessible and high construction density areas [3] as wireless signal needs frequent line of sight for passing. In this purpose, bandwidth coverage of wireless $5 \mathrm{G}$ micro cell is decreased. To solve the limitation, hybrid communication can play a vital role. As the electrical transmission line reaches everywhere, BPL can provide the proper bandwidth coverage where the wireless signal can't pass sufficiently. The feature can also understand from the Fig. 2. Both communication work as alternate to other.

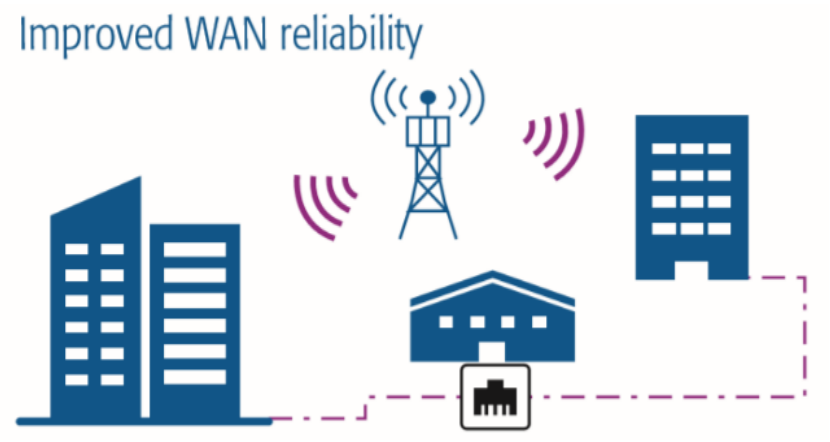

Fig. 1. Communication Reliability [3].

\section{Improved bandwidth coverage}

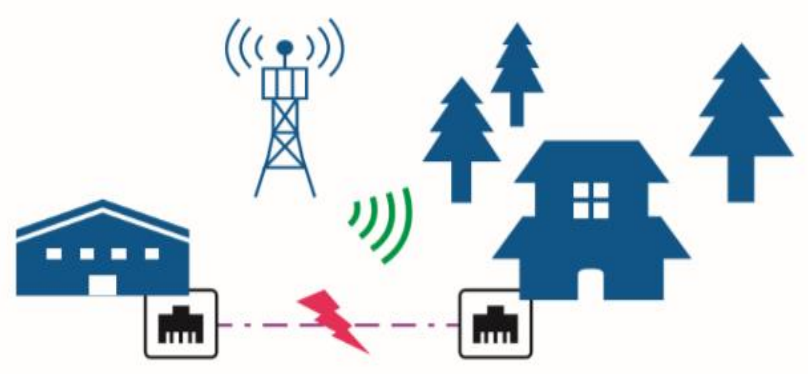

Fig. 2. Bandwidth Coverage [3]. 


\section{Throughput Capacity}

The throughput capacity is one of the most concerning issues in communication. Users need better data rate continuously [3]. However, wireless communication has some challenges, especially in $5 \mathrm{G}$. In the future, a plethora of machines and devices will connect with the internet via technology. So, it has the chance to decrease the data speed. To stabilize the regular throughput capacity in a critical situation and increase the throughput in regular time, hybrid communication can aggregate the bandwidth from both ends. The feature has displayed in Fig. 3.
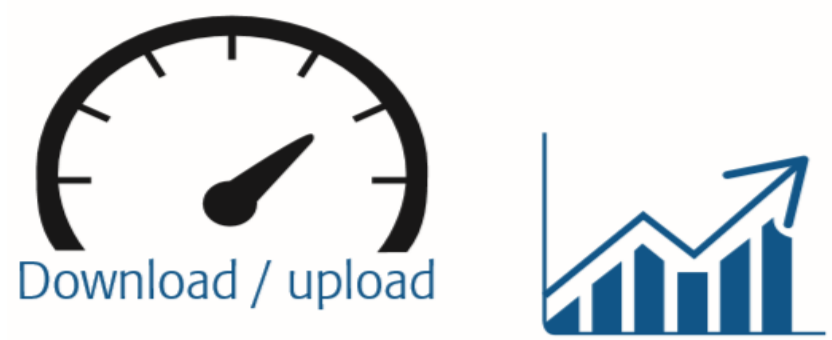

\section{Increased access capacity}

Fig. 3. Improving throughput Capacity [3].

\section{Cost-Effectiveness}

There are so many options to develop a hybrid communication with wireless communication such as optical fiber, DSL, or satellite technology. However, Broadband over Power Line is the most cost-effective than other technologies. The technology doesn't need extra infrastructure, and electrical line can be used for it. Moreover, AT\&T has already developed the prototype MIMO antenna for serving the internet via BPL technology [12]. The antenna will be placed on the electrical pole near the users' home. So, it is more convenient to serve data combinedly from a common hybrid small cell.

\section{BASIC COMPOMEMTS OF THE PROPOSED HYBRID COMMUNICATION MODEL}

The hybrid network connects the Wireless and Broadband over Power Line communication simultaneously. The architecture has three main parts, and those are described below.

\section{A. Hybrid Small Cell (HSC)}

Hybrid Small Cell resides at the customer premises [1]. The cell allows to access both channel for the users simultaneously. It can perform for both uplink and downlink. When an uplink request is received from the user devices, it will take the confirmation of the access path from the traffic distribution control unit. It retrieves the data of BPL from the electrical power line and wireless data. The small cell will be designed for aggregating wireless and BPL technology. AT\&T has already designed a MIMO antenna for BPL Wi-Fi.

\section{B. Traffic Distribution Control Unit (TDCU)}

The unit is responsible for forwarding the users' information over the wireless and BPL network access path
[3]. It provides load balancing, scheduling and congestion control of the network. The feedback information from the unit helps to take decisions on distributing uplink traffics so that it acts as an uplink data controller.

\section{Hybrid Access Gateway (HAG)}

The gateway is a logical function in the architecture. It is placed in the operation's core network and performs data controlling mechanisms for simultaneous use of wireless and BPL network. It allows data passing to/ from outside the network or internet. It permits the downstream data to pass through a single path by using a single path protocol.

\section{COMmunication Flow OF the Model}

Wireless and Broadband over Power Line both will connect with the network simultaneously. In Fig. 4, the hybrid model has been demonstrated. However, the user's data will pass through a single path at a time. The mechanism will be provided by using single access protocol in HSC and HAG. In the architecture, users are connected with the HSC, and the connection will be via wireless communication. When the HSC unit gets requests from the users, then it checks the availability of the access paths for data passing by using the traffic distribution control unit. TDCU allows the data to pass through an access path, and the data shifts to the HAG. Then HAG permits the data to go to the internet. In the same way, when a data request comes from the other network or internet, HAG checks the availability of the access channels by using a single channel protocol [1] and provides the permission to pass through a single channel.

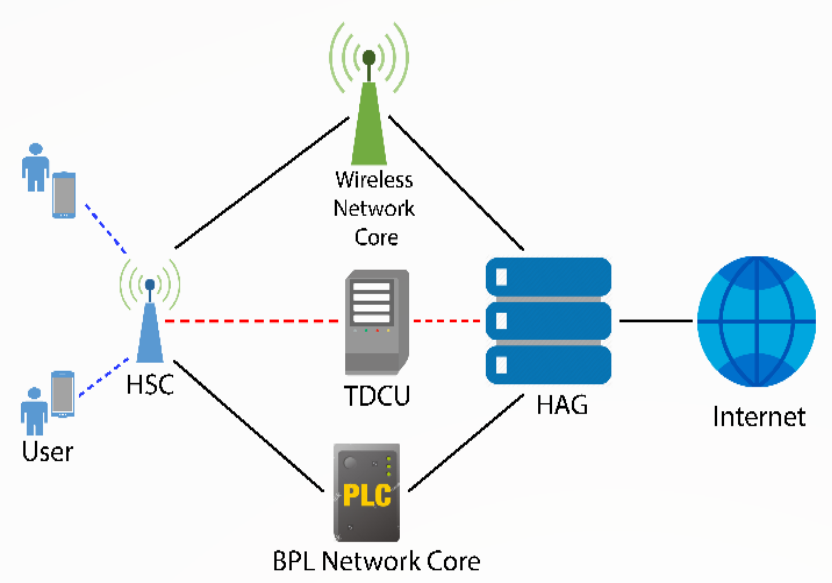

Fig. 4. Hybrid Wireless/BPL Communication Model.

\section{Throughrut CALCULATION}

\section{A. Throughput Calculation of Broadband over Power Line}

Broadband over Power Line can be an alternative solution for hybrid communication. It is cost-effective, and its advancement so far is significant. Moreover, the installation period is short. For the sake of those reasons, deployment of the technology in any area is more convenient than other wired communication technologies. In project AirGig, MIMO antenna will play the role of the extractor and serve $5 \mathrm{G}$ via BPL technology [12]. 
In Broadband over Power Line technology, the data signal passes through the conventional electrical medium and low voltage line with the $60 \mathrm{~Hz} / 50 \mathrm{~Hz}$ electrical signal simultaneously. So, there is no possibility to make interference between $\mathrm{mm}$ wave and regular electrical signal.

In this research, $28 \mathrm{GHz}$ has been proposed as the operating frequency. Four types of noise have been presented in broadband over power line. First of them is coloured background noise. The source of the noise is multiple low power loads such as motor, fan, etc. [12]. The next noise is known as narrow-band noise, and it is created from broadcasting stations like television and radio station [12]. The last two noises are synchronized impulse noise and asynchronized impulse noise [12]. Those have significant effects on data signal. Circuit breaker, thyristor is the source of the noises.

Power Spectral density (PSD) of Coloured Background Noise [12] is given below:

$\mathrm{N}_{\mathrm{CBN}}=\mathrm{N}_{0}+\mathrm{N}_{1} \cdot \mathrm{e}^{-(\mathrm{f} / \mathrm{f} 1)}$

In equation $1, \mathrm{~N}_{0}$ and $\mathrm{N}_{1}$ indicate the constant noise density. The value of $\mathrm{N}_{0}$ and $\mathrm{N}_{1}$ are respectively -35 and $35 . \mathrm{f}$ refers the carrier frequency and $\mathrm{f} 1$ indicates the exponential function.

In Fig. 5, the green line carries the value of PSD for home purposes, and the red line carries the value for industrial purposes. In this research, the value of PSD for home is exploited, and it is $-33 \mathrm{~dB}$.

Moreover, the power spectral density of impulse noise is expressed as [11].

$\left.\mathrm{P}(\mathrm{t})=(\mathrm{u}(\mathrm{t}))-\mathrm{u}\left(\mathrm{t}-\mathrm{T}_{\mathrm{D}}\right)\right) \cdot \sum_{i=0}^{N d-1} \frac{A i}{N d} \cdot e^{-\alpha i|t|} \cdot e^{-j 2 \pi f t}$

In equation $2, \mathrm{u}(\mathrm{t})$ is the step function. $\mathrm{N}_{\mathrm{d}}$ is the demand of sinusoid the value of which is $3, \mathrm{~T}_{\mathrm{D}}$ refers to the duration of the impulse. $\alpha_{i}$ has a constant value $0.3 \times 106$. In Fig. 6 , range of the periodic impulse noise is from $0.04 \mathrm{~V}$ to $0.06 \mathrm{~V}$.

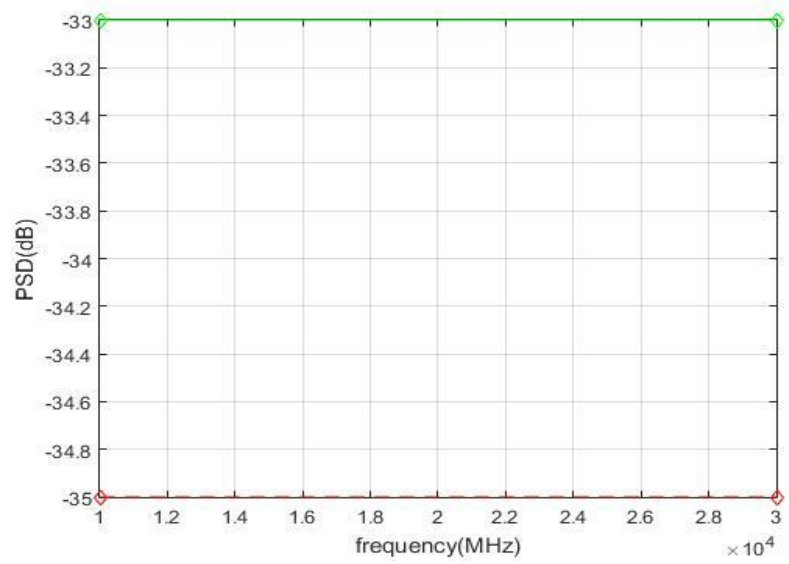

Fig. 5. PSD of Coloured Background Noise for $28 \mathrm{GHz}$.

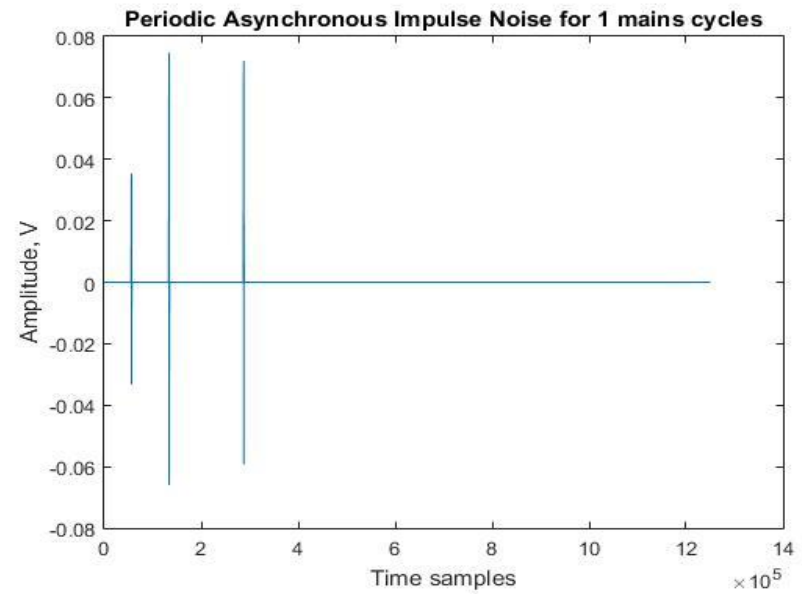

Fig. 6. The Periodic Impulse Noise in Broadband over Power Line.

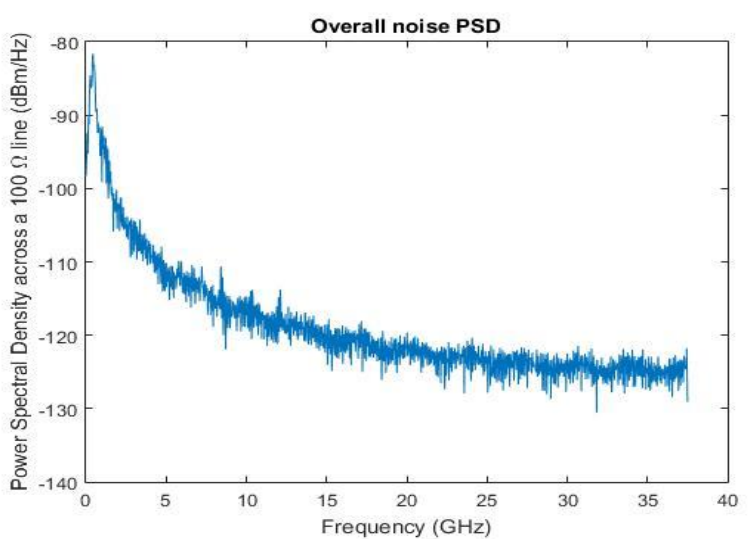

Fig. 7. Total Power Spectral Density of BPL Noises.

The total power spectral density (PSD) of all four noises together in Broadband over Power Line is shown in Fig. 7. The value of the total PSD of BPL in $28 \mathrm{GHz}$ is measured to be $-125 \mathrm{~dB}$ by using the Cumulative Power Line Noise function.

The cycle of $28 \mathrm{GHz}$ signal is assumed in this research. The power of the required BPL data signal is given below [14].

$\mathrm{P}_{\mathrm{x}}=\lim _{N \rightarrow \infty}\left(\frac{1}{2 N+1}\right) \cdot \sum_{n=-N}^{n}\left|x_{n}\right|^{\wedge} 2$

In this equation $x_{n}$ refers to the data signal mathematically and it is defined as.

$\mathrm{x}_{\mathrm{n}}=\mathrm{A} \sin 2 \pi \mathrm{ft}$

Amplitude of the data signal is denoted as A in (4) and it is 1. Moreover, in equation (3), $\mathrm{N}$ refers to the length of signal. The power of the signal is measured to be $22 \mathrm{w}$. The matlab simulation of the power measurement has been shown in Fig. 8. 

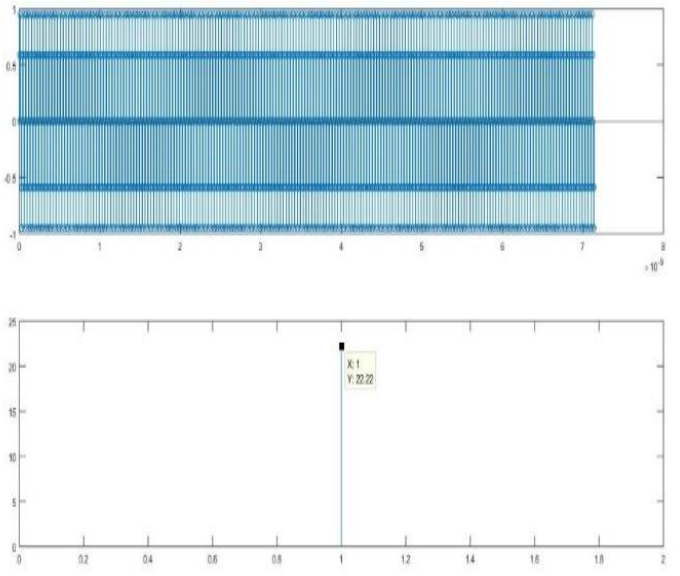

Fig. 8. Power of the Broadband over Power Line Signal in 28GHz.

The Shannon limit theory is exploited to measure the throughput of broadband over power line. The formula of Shannon limit is given below [11]:

$\mathrm{C}=\mathrm{Blog}_{2}\left(1+\frac{P x}{P(t)+N(C B N)}\right)$

$=7.84 \mathrm{Gbps}$

\section{B. Throughput Calculation of Wireless Communication}

Wireless $5 \mathrm{G}$ will deliver hundred times faster data speed than $4 \mathrm{G}$ communication [1]. In this research, we have shown the throughput rate of $5 \mathrm{G}$ in $28 \mathrm{GHz}$ frequency. The operating frequency can provide $300 \mathrm{MHz}$ bandwidth [13].

$\mathrm{T}_{\mathrm{p}}=10^{-6} \cdot \sum_{j=1}^{j}\left(v_{\text {Layer }}^{(j)} \cdot Q_{m}^{(j)} \cdot f^{(j)} \cdot R_{\max } \cdot \frac{N_{P R B}^{B W(j), \mu} \cdot 12}{T_{s}^{\mu}} \cdot(1-\right.$ $\left.\left.O H^{(j)}\right)\right)$

The equation (6) is formulated by 3GPP TS 38.306 [13] and it is in FR2 range. The maximum no. of $\mathrm{v}_{-} \operatorname{Layer}^{\wedge}((\mathrm{j}))$ is 4 and maximum modulation order $\left.\mathrm{Q} \_\mathrm{m}^{\wedge}(\mathrm{j})\right)$ is 8 (256 QAM) [14]. In equation (6), scaling factor $\mathrm{f}^{\wedge}((\mathrm{j}))$ is 1 and $\mathrm{NR} \mu=3$ (maximum). For OFDM, symbol duration $\mathrm{T}$ is $\_\mathrm{s}^{\wedge} \mu=10^{\wedge}(-$ $3) /\left(14.2^{\wedge} \mu\right)$. The important parameter of the equation, bandwidth $(\mathrm{BW})=300 \mathrm{MHz}$ as $3 \mathrm{GPP}$ TS 38.306 policy [13]. PRB refers to the number of resource blocks for the particular bandwidth. In this research, the data sharing mode is TDD for both uplink and downlink.

The formula of PRB is expressed as [15]

$$
\begin{aligned}
& \mathrm{PRB}=\frac{(\text { Channel BW}-2 \times \text { Guard Bandwidth })}{\text { Single Resource Block Size }} \\
& =194.63 \text { 195 }
\end{aligned}
$$

A single resource block for $\mu=3$ is $1440 \mathrm{KHz}$ [15], and the guard band is 9860 [13].

By using the equation (6), the throughput is $5.45 \mathrm{Gbps}$ for downlink and 998Mbps - 1 Gbps for uplink, respectively.

In Fig. 9, the throughput of both wireless and Broadband over Power Line has been displayed. In Fig. 9, it is shown that Broadband over Power Line technology has good possibility to align with wireless for establishing a hybrid network.

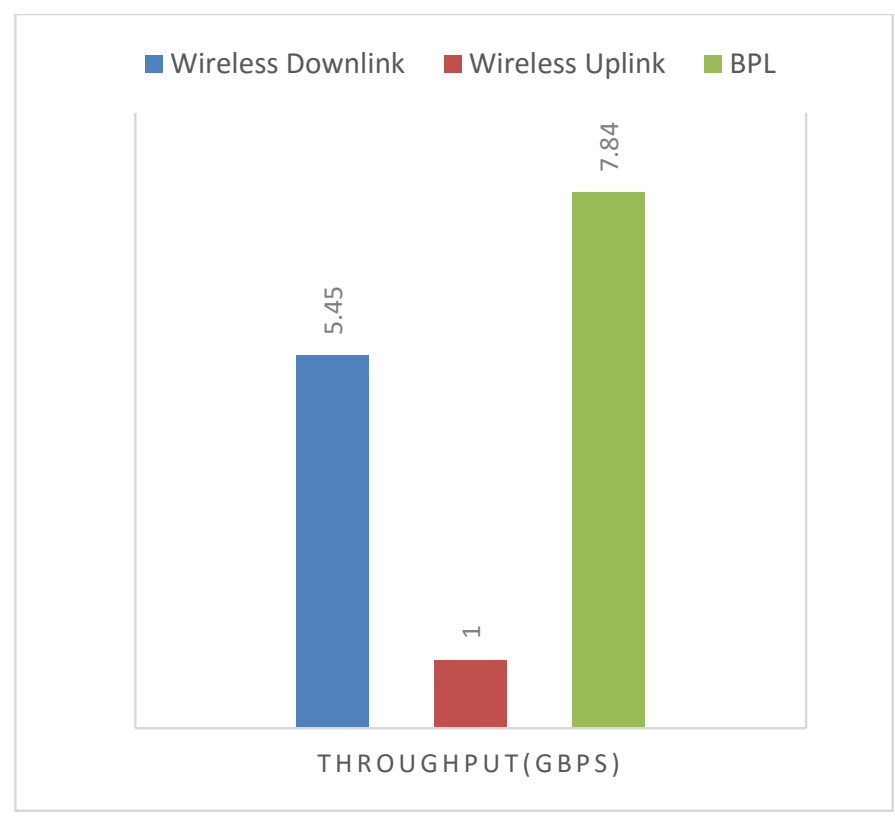

Fig. 9. Throughput of both Technologies.

\section{CONCLUSION}

Hybrid communication can confirm the continuity of data flow to the end devices, and it will also provide service in the disaster period. As it manages a dual source, robust throughput can be achieved in general time. The main challenge of the model is data traffic management. However, it can be recovered by switching the operation of a small cell. In future research, the model will be designed for $6 \mathrm{G}$ communication as $6 \mathrm{G}$ needs robust data and some researchers have already proposed terahertz frequency for $6 \mathrm{G}$. However, the terahertz frequency is not suitable for an average distance, and hardware design will be difficult for it. So multiple communication aggregation can be a new solution for $6 \mathrm{G}$ technology.

\section{ACKNOWLEDGMENT}

The work was supported by IIUC Research Grants [Grant Number IRG 180108]. The authors express their gratitude to the Center for Research and Publication [CRP] of International Islamic University Chittagong [IIUC] for funding the project.

\section{REFERENCES}

[1] C. K. Agubor, I. Akwukwuegbu, and M. Olubiwe, "A comprehensive review on the feasibility and challenges of millimeter wave in emerging 5G mobile communicatio," Advances in Science, Technology and Engineering Systems Journal Vol. 4, No. 3, 138-144 (2019).

[2] T. C. Chuah, Y. L. Lee, "QoS-aware Cross-Layer optimization of hybrid DSL-LTE access networks.", IEEE SYSTEMS JOURNAL, June 29, 2019.

[3] K. Samdanis, F. Leitao, S. Oechsner, J. R. I. Riu, R. D. C. Ros and G. Fabregas, "From interworking to hybrid access systems and the road toward the next-generation of fixed-mobile convergence," in IEEE Communications Standards Magazine, vol. 1, no. 1, pp. 36-43, March 2017.

[4] M. Condoluci et al., "Fixed-mobile convergence in the $5 \mathrm{~g}$ era: from hybrid access to converged core," in IEEE Network, vol. 33, no. 2, pp. 138-145, March/April 2019. 
[5] M. A. Hasabelnaby, H. A. I. Selmy and M. I. Dessouky, "C-RAN availability improvement using parallel hybrid fso/mmw $5 \mathrm{~g}$ fronthaul network," 2018 International Japan-Africa Conference on Electronics, Communications and Computations (JAC-ECC), Alexandria, Egypt, 2018, pp. 130-133.

[6] A. T. Pham, P. V. Trinh, V. V. Mai, N. T. Dang and Cong-Thang Truong, "Hybrid free-space optics/millimeter-wave architecture for 5G cellular backhaul networks," 2015 Opto-Electronics and Communications Conference (OECC), Shanghai, 2015, pp. 1-3.

[7] A. Abdelsalam, M. Luglio, C. Roseti, and F. Zanpongnaro, " Analysis of bandwidth aggregation techniques for combined use of satellite and XDSL broadband link", in International Journal of Satellite Communications and Networkiing, vol 37, no 2, pp. 1-15, March 16, 2018.

[8] O. Ulgen, U. Ozmat and E. Gunaydin, "Hybrid implementation of millimeter wave and visible light communications for 5G networks," 2018 26th Telecommunications Forum (TELFOR), Belgrade, 2018, pp. $1-4$.

[9] P. T. Dat, A. Kanno, and K. Inagaki1, “ Hybrid optical wirelessmmwave: ultra highspeed indoor communications for beyond 5G “ 2019 IEEE INFOCOM Poster, pp. 1003-104.

[10] M. Z. Chowdhury, M. K. Hasan, M. Shahjalal, M. T. Hossan and Y. Min Jang, "Optical wireless hybrid networks for $5 \mathrm{~g}$ and beyond communications," 2018 International Conference on Information and Communication Technology Convergence (ICTC), Jeju, 2018, pp. 709712 .
[11] G. Prasad, L. Lampe and S. Shekhar, "In-band full duplex broadband power line communications," in IEEE Transactions on Communications, vol. 64, no. 9, pp. 3915-3931, Sept. 2016.

[12] M. W. Ullah, M.A Rahman, M.M.A. FaIsal and A. Gafur, "Feasibility of power line communication in bangladesh and analyzing the power spectral density of colored background noise, International Conference on Industrial Engineering and Operations Management Washington DC, USA, pp. 1117-1124, September 27-29, 2018.

[13] "Bandwidth allocation sheet", Available: file:///F:/Smart\%20Grid\%20 and $\% 20$ Hybrid\%20Communication $\% 205 \mathrm{G} /$ Hybrid\%20Communication \%20Paper/5G\%20_\%20ShareTechnote.html, [Accessed: 31 ${ }^{\text {st }}$ March 2020].

[14] “ $5 \mathrm{G}$ wireless communication throughput formula" Available: file:///F:/Smart\%20Grid\%20and\%20Hybrid\%20Communication\%205G/ Hybrid\%20Communication\%20Paper/5G\%20New\%20Radio\%20Techn ology\%20Throughput\%20Calculation.html, [Accessed: 31 ${ }^{\text {st }}$ March 2020].

[15] "5G throughput calculator" Available: file:///F:/Smart\% 20Grid\%20and $\% 20$ Hybrid\%20Communication\%205G/Hybrid\%20Communication $\% 20$ Paper/5G\%20NR\%20Throughput\%20calculator\%20_\%205GTools.com.html, [Accessed: $31^{\text {st }}$ March 2020]. 